\title{
Onlineprüfung Sachverstand Strahlenschutz
}

M. Otto, W. Zeller

Knapp die Hälfte aller Kandidaten hat die Prüfung «Sachverstand Strahlenschutz» online absolviert, von den 2004 abgelegten Prüfungen waren es sogar vier Fünftel. Die Onlineprüfung hat damit die Erwartungen seiner Auftraggeber deutlich übertroffen.

Korrespondenz:

Dr. Marc Otto

pnn pharma nation network ag

Technopark Zürich

Technoparkstrasse 1

CH-8005 Zürich

Internet: http://pnn.ethz.ch

Dr. Werner Zeller

Bundesamt für Gesundheit

Abteilung Strahlenschutz

CH-3003 Bern

\section{Ausgangslage}

«Ärztinnen und Ärzte, die vor dem 1. Oktober 1994 eine Bewilligung zum Betreiben einer Röntgenanlage erhalten haben, gelten bis zum 30. September 2004 als Sachverständige. Wird die Röntgenanlage nach diesem Datum weiter betrieben, muss der Sachverstand in einer Prüfung nachgewiesen werden.» So lautete die Ausgangslage, welche viele niedergelassene Ärztinnen und Ärzte dazu brachte, sich das Kompendium für ärztliche Strahlenschutzsachverständige zu behändigen und für die Prüfung anzumelden.

«Um den Ärztinnen und Ärzten das Ablegen der Prüfung zu erleichtern, bietet das BAG auf Anregung der FMH von Anfang Februar bis Ende Juni 2004 die Möglichkeit, die Prüfung vom eigenen PC aus online zu absolvieren. Alles, was es dazu braucht, ist ein Internetzugriff und eine gültige E-Mail-Adresse. Mit der Abwicklung der Prüfung wurde die Firma pnn ag beauftragt, eine Spin-off-Gesellschaft der ETH Zürich, die sich auf medizinische Onlinefortbildung spezialisiert hat. Als Grundlage für die Vorbereitung dient das Kompendium für ärztliche Strahlenschutzsachverständige.» So wurde ein neuartiges Projekt angekündigt: eine der ersten verbindlichen Onlineprüfungen Europas.

\section{Onlineprüfung}

Seit Anfang Februar 2004 konnten sich Ärztinnen und Ärzte via Internet für die Onlineprüfung anmelden. Das Bundesamt für Gesundheit, BAG, hat anhand der eingegebenen Daten überprüft, ob die Bewerbenden für die Prüfung überhaupt zugelassen werden können. Wenn ja, wurde eine achtstellige Zufallszahl als Zugangscode verschickt. Zusammen mit dem Passwort, das bei der Anmeldung jeder Teilnehmende festgelegt hatte, verschaffte der Zugangscode dann Zugriff auf die Onlineprüfung, welche innert
60 Minuten bearbeitet werden musste. Die Prüfung galt als bestanden, wenn $60 \%$ der Fragen richtig beantwortet worden sind.

\section{Halbautomatischer Ablaufprozess}

Die Prüfungen wurden vollautomatisch ausgewertet und die Resultate zusätzlich manuell überprüft. War ein Prüfungsresultat vom BAG bestätigt, wurden automatisch alle nötigen Dokumente generiert und die Verfügung dem Bundesamt zur Unterschrift zugestellt.

Die Onlineprüfung war so ausgelegt, dass die Fragen in beliebiger Reihenfolge bearbeitet und bereits gegebene Antworten korrigiert werden konnten. Waren keine Änderungen mehr nötig, konnten die Teilnehmenden die Prüfung mit Klick auf einen Button abgeben. Eine Sicherheitsabfrage wies dann allenfalls auf noch nicht beantwortete Fragen hin. Wurde sie bestätigt, wurde die Prüfung abgeschlossen und zur automatischen Korrektur weitergeleitet.

War die Prüfungsdauer von 60 Minuten erreicht, wurde die Prüfung automatisch abgeschlossen. War während der Prüfung beispielsweise die Verbindung ins Internet zusammengebrochen, konnte die Prüfung nach telefonischer Rücksprache mit dem BAG innert 24 Stunden wiederaufgenommen und fertig beantwortet werden.

Um sich mit der Navigation vertraut $\mathrm{zu}$ machen, wurde den Teilnehmenden eine Onlinetestprüfung angeboten. Deren MC-Fragen hatten inhaltlich natürlich nichts mit Strahlenschutz zu tun.

\section{Technische Umsetzung}

Die technische Umsetzung war darauf ausgerichtet, dass man die Onlineprüfung auch mit älteren Computern und schmalbandigen Modems besuchen kann. Es wurde deshalb auch darauf verzichtet, dass spezielle Software oder Plug-ins für die Prüfung installiert werden müssen. Dieser Ansatz hatte sich schon beim CME des Swiss Medical Forum bewährt, der Onlinefortbildung hinter der pnn ag gemeinsam mit dem Schweizerischen Ärzteverlag EMH steht. 
Als MC-Fragetypen wurden A, E und KPrim eingesetzt, die Fragetexte wurden vom AUM der Universität Bern verfasst und validiert. Die Fragen für jede Prüfung wurden zufällig aus einem grossen Fragepool zusammengestellt, wobei ein Blueprint dafür sorgte, dass die Anteile der verschiedenen Fragetypen, der behandelten Themen und dadurch auch der Schwierigkeitsgrad der Prüfung immer dieselben waren.

\section{Abbildung 1}

Altersstruktur der Teilnehmer.

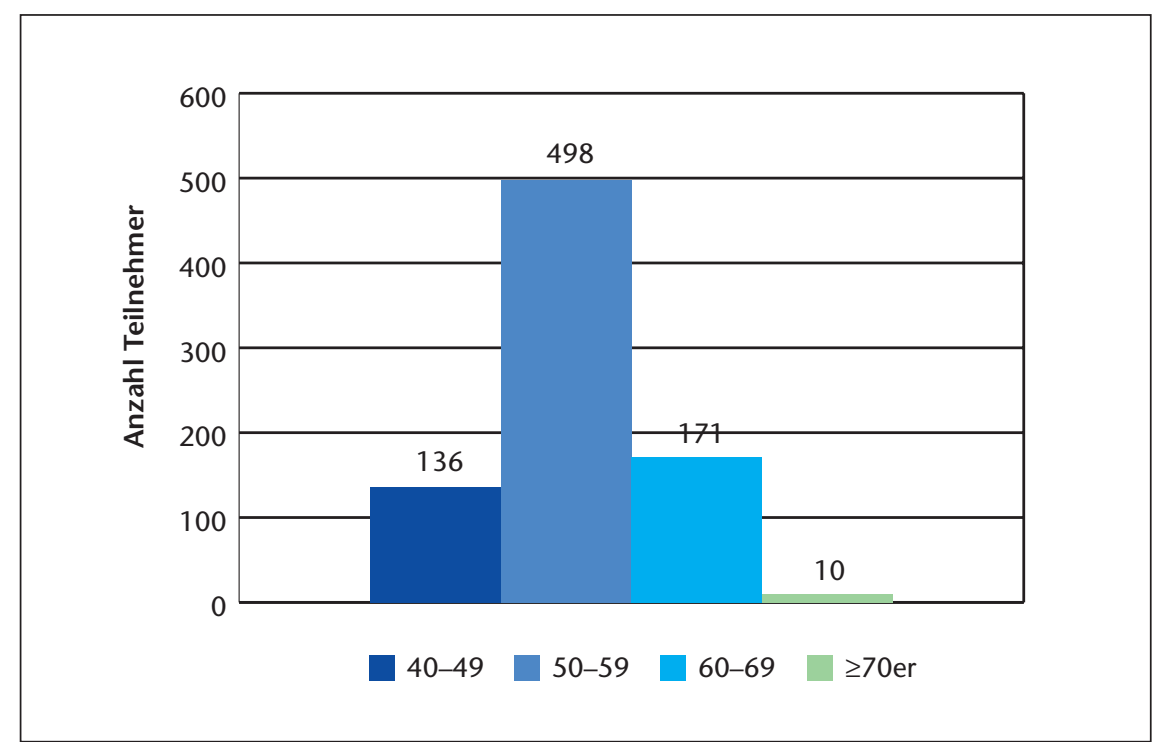

Abbildung 2

Geographische Herkunft der Teilnehmer.

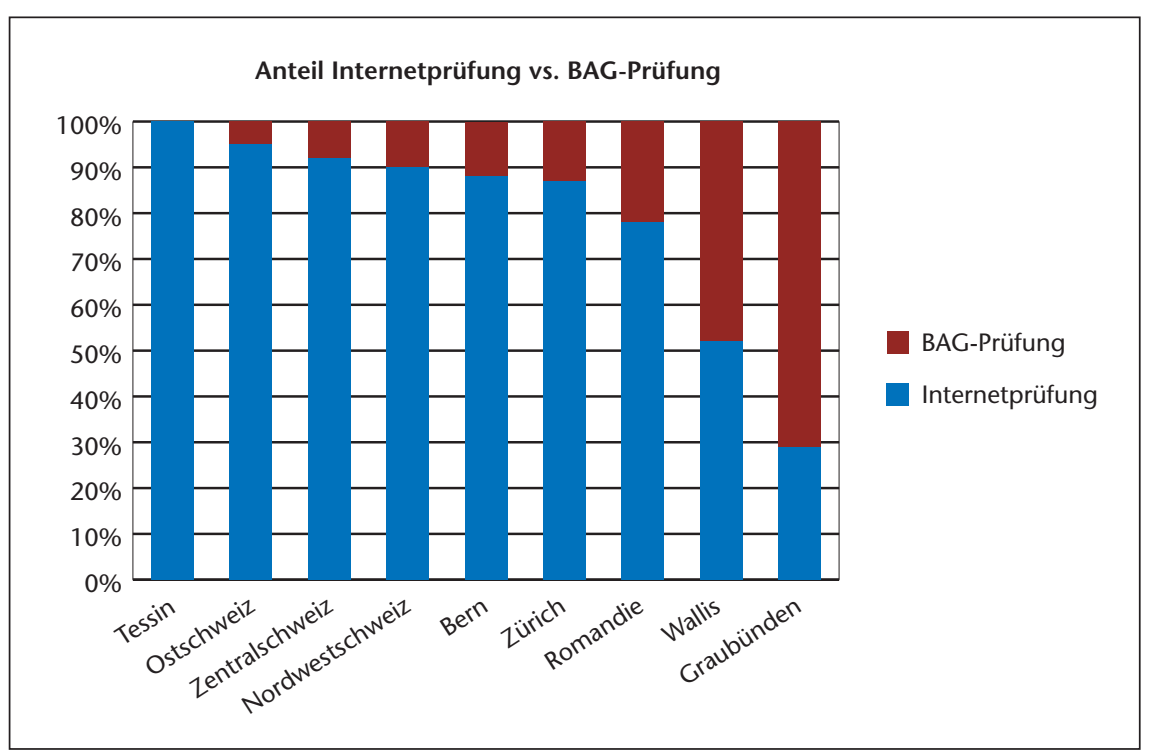

Anmerkung:

- $\quad$ VS und GR weisen einen geringeren Anteil von Internetprüfungen auf, da jeweils durch die kantonale Ärztegesellschaft eine Prüfung organisiert wurde.

- $\quad$ TI: nur fünf Absolventen, welche fast alle einen Sachverständigenkurs besuchten.
Beim Einwählen in die Prüfung wurden neben dem Teilnehmenden auch sein Computer technisch identifiziert. Damit der Prüfungsverlauf jedes Teilnehmenden immer rekonstruierbar blieb, wurde zudem jeder Klick zusammen mit einem «timestamp» festgehalten. Nur so konnte eine Prüfung nach technischem Unterbruch weitergeführt und die Prüfungsresultate zuverlässig überprüft werden. Zudem wurde mit automatischem und halbautomatischem Monitoring der gesamte Prüfungsablauf vom BAG und von der pnn ag feinmaschig überwacht.

Die Überwachung der Prüfung wurde dadurch erleichtert, dass alle notwendigen Informationen in einem geschützten Raum via Internet zugänglich waren. Dies hat die Zusammenarbeit vom Usersupport der pnn ag mit dem Team des BAG erleichtert, so dass der Ablaufprozess von der Anmeldung bis zum Inkasso der Prüfungsgebühren effizient und rasch funktionierte, obwohl die beiden Organisationen mehr als $100 \mathrm{~km}$ voneinander weg angesiedelt sind.

Um die Daten vor fremdem Zugriff zu schützen, wurde harte Kryptographie sowohl für die Übertragung wie auch für die Speicherung auf dem Server eingesetzt. Der Server selbst war redundant ausgelegt und in einem sicheren Rechenzentrum installiert.

\section{Erfahrungen}

Die Onlineprüfung wurde von 815 Teilnehmenden absolviert. Das sind $84 \%$ aller, die im Jahr 2004 die Prüfung abgelegt haben, oder 46\% derjenigen, die die Prüfung zwischen 2001 und 2004 abgelegt haben. Die Teilnehmenden, welche in den 1940er Jahren geboren wurden, machten $49 \%$ aller Prüflinge aus, gefolgt von $40 \%$ der 1950 er, $17 \%$ der 1960 er und $5 \%$ der 1930er Jahrgänger.

Von den 815 Teilnehmenden mussten 20 die Prüfung wiederholen, da sie im ersten Durchgang die erforderliche Punktzahl nicht erreicht hatten.

Der telefonische Benutzersupport hat in rund 150 Arbeitsstunden über 500 Anrufe bearbeitet. Drei Teilnehmer konnten sich mit ihrem PC trotz allem nicht in die Prüfung einwählen, so dass sie die Prüfung von einem anderen Gerät aus absolviert haben. Ein Teilnehmer konnte die Schrift am Bildschirm nicht lesen, so dass er die Prüfung im traditionellen Rahmen abgelegt hatte.

Viele Ärztinnen und Ärzte haben sich nach bestandener Prüfung per Telefon und E-Mail gemeldet. Die folgenden zwei Zitate sind zufällig gewählt, zeigen aber die positive Resonanz: 
- AK: «Im übrigen möchte ich Euch ein Kompliment machen für die Art der Prüfung bzw. Einführung: auch für ein älteres, normal intelligentes Wesen in kurzer Zeit verstehbar.»

- BM: «Ich habe heute die erwähnte Prüfung absolviert und möchte mich für die klaren Anweisungen, die übersichtliche optische Darstellung und die m.E. fairen Fragen ganz herzlich bei den Verantwortlichen bedanken!»

\section{Fazit}

Obwohl die Prüfung zum Nachweis des Strahlenschutzes anfänglich zu vielen, teilweise hitzigen Diskussionen Anlass gegeben hat, ist die Onlineprüfung von der Ärzteschaft sehr positiv aufgenommen worden. Das zeigen die grosse Zahl der Absolventen und die vielen positiven Reaktio- nen. Das im Vergleich mit den Nutzern des SwissMedical-Forum-CME deutlich höhere Durchschnittsalter der Teilnehmer der Onlineprüfung scheint deren Akzeptanz kaum negativ beeinflusst zu haben. Zum Erfolg der Onlineprüfung in Strahlenschutz hat sicherlich auch beigetragen, dass viel in den telefonischen Benutzersupport und die einfache Benutzerführung investiert worden ist. Zudem wurde die Frist, in welcher die Prüfung online abgelegt werden konnte, zweimal um jeweils einen Monat verlängert. Last, but not least konnte die Onlineprüfung nur deshalb so schnell umgesetzt und so problemlos abgewickelt werden, da sie vom BAG akribisch geplant worden ist, das AUM fundiert evaluierte MC-Fragen zur Verfügung gestellt hat und alle Parteien festgestellte kleinere Probleme immer schnell und unkompliziert beheben und notfalls rasch neue Lösungsansätze implementieren konnten. 